\title{
Comparison of laparoscopic versus conventional open surgical staging procedure for endometrial cancer
}

\author{
Tae Wook Kong, Kyung Mi Lee, Ji Yoon Cheong, Woo Young Kim, Suk-Joon Chang, \\ Seung-Chul Yoo, Jong-Hyuck Yoon, Ki-Hong Chang, Hee-Sug Ryu \\ Department of Obstetrics and Gynecology, Ajou University School of Medicine, Suwon, Korea
}

\begin{abstract}
Objective: The aim of this study was to compare the surgical outcomes of laparoscopic surgery and conventional laparotomy for endometrial cancer.

Methods: A total of 104 consecutive patients were non-randomly assigned to either laparoscopic surgery or laparotomy. All patients underwent comprehensive surgical staging procedures including total hysterectomy, bilateral salpingo-oophorectomy, and pelvic/para-aortic lymphadenectomy. The safety, morbidity, and survival rates of the two groups were compared, and the data was retrospectively analyzed.

Results: Thirty-four patients received laparoscopic surgery and 70 underwent laparotomy. Operation time for the laparoscopic procedure was $227.0 \pm 28.8$ minutes, which showed significant difference from the 208.1 \pm 46.4 minutes $(\mathrm{p}=0.032)$ of the laparotomy group. The estimated blood loss of patients undergoing laparoscopic surgery was $230.3 \pm 92.4 \mathrm{~mL}$. This was significantly less than that of the laparotomy group $(301.9 \pm 156.3 \mathrm{~mL}, \mathrm{p}=0.015)$. The laparoscopic group had an average of 20.8 pelvic and 9.1 para-aortic nodes retrieved, as compared to 17.2 pelvic and 8.5 para-aortic nodes retrieved in the laparotomy group. There was no significant difference $(p=0.062, p=0.554)$. The mean hospitalization duration was significantly greater in the laparotomy group than the laparoscopic group (23.3 and 16.4 days, $\mathrm{p}<0.001$ ). The incidence of postoperative complications was $15.7 \%$ and $11.8 \%$ in the laparotomy and laparoscopic groups respectively. No statistically significant difference was found between the two groups in the survival rate.

Conclusion: Laparoscopic surgical staging operation is a safe and effective therapeutic procedure for management of endometrial cancer with an acceptable morbidity compared to the laparotomic approach, and is characterized by far less blood loss and shorter postoperative hospitalization.
\end{abstract}

Key Words: Endometrial cancer, Laparoscopic surgical staging, Laparotomy

\section{INTRODUCTION}

Endometrial cancer is the most common gynecologic malignancy, with increasing trends particularly among postmenopausal women, and is diagnosed at an early stage, i.e., when the malignancy is confined to the uterus in $75 \%$ of cases. Changes in reproductive behavior and increased rate of obesity may partially attribute to the increase. ${ }^{1,2}$

The mainstay of initial treatment is a comprehensive surgical staging operation, which is usually curative and includes abdominal exploration, pelvic peritoneal cytology, total hys-

Received March 29, 2010, Revised May 13, 2010,

Accepted May 24, 2010

\section{Correspondence to Suk-Joon Chang}

Department of Obstetrics and Gynecology, Ajou University School of Medicine, San 5 Woncheon-dong, Youngtong-gu, Suwon 443-721, Korea

Tel: 82-31-219-5251, Fax: 82-31-219-5245

E-mail: drchang@ajou.ac.kr terectomy, bilateral salpingo-oophorectomy and pelvic/paraaortic lymphadenectomy. ${ }^{2}$ Since Childers and Surwit first proposed laparoscopic surgical staging as an alternative for early endometrial cancer, several studies have shown that this approach was an effective and economically efficient alternative to open surgery. ${ }^{3-7}$ It is known that the laparoscopic approach is associated with a shorter hospitalization, faster recovery, lower risk of thromboembolic complications, and postoperative infections.

Obesity is closely associated with medical co-morbidities including diabetes, cardiovascular disease, and osteoarthritis, and also a major risk factor for endometrial cancer. Patients with endometrial cancer often are both obese and elderly, which makes comprehensive laparoscopic surgical staging difficult, and surgeons often prefer to perform a laparotomy. ${ }^{6-8}$ A recent study reported that $68 \%$ of women with early-stage endometrial cancer are obese. ${ }^{9}$ Other factors such as adhesive disease, large uteri, fatty mesentery, impaired tolerance to Trendelenburg position, and surgical skills have lim- 
ited widespread use of this approach.

Two meta-analyses based on results of 17 prospective and retrospective studies demonstrated that lower postoperative complications were associated to laparoscopy, and that there was no significant difference between the laparoscopic and laparotomic approaches to endometrial cancer in disease-free and overall survival. ${ }^{9,10}$ In 2009 , Walker et al. ${ }^{11}$ published the result of one randomized controlled trial (LAP2) by the Gynecologic Oncology Group (GOG). They concluded that laparoscopy is an acceptable alternative to laparotomy for the comprehensive surgical staging of uterine cancer. Despite these established short-term advantages of the laparoscopic surgery, there is no general agreement about the survival benefit of laparoscopic surgical staging procedures. The main reason for this is that survival data from well designed prospective studies are not sufficient, although recently, initial survival data from LAP2 was reported at the 41st annual meeting of the Society of Gynecologic Oncologists (SGO), demonstrating identical 3-year overall survival between laparotomy and laparoscopy. ${ }^{12}$

The current study aimed to compare surgical staging procedures with laparoscopy and laparotomy for the treatment of endometrial cancer.

\section{MATERIALS AND METHODS}

One-hundred four consecutive patients with endometrial cancer who were thought to be clinical stage I disease were treated from March 2006 to February 2009 at Ajou University Hospital. All patients were histologically confirmed as endometrioid adenocarcinoma, papillary serous carcinoma, or mixed carcinoma, and were included in the study. All cases of sarcomas and of presumed advanced-stage disease were excluded. Approval was given by the Ajou University Hospital Institutional Review Board.

All eligible patients underwent total hysterectomy, bilateral salpingo-oophorectomy, peritoneal washing cytology, pelvic lymphadenectomy, and para-aortic lymphadenectomy. All procedures were conducted via laparotomy or laparoscopy, and the decision as to which patients received laparotomy or laparoscopy was determined by the surgeon's and the patient's preference. Some surgeons preferred to perform staging procedures laparoscopically, whereas others preferred the laparotomic approach. Patients were offered the laparoscopic procedure according to the age, parity, body mass index (BMI), and medical or surgical history, and were informed about the possibility of conversion from laparoscopy to laparotomy, and all the participants gave written informed consent. Patients with documented significant cardiopulmonary disease were refused a laparoscopic approach after consultation with a member of the anesthesiology team. All patients underwent preoperative imaging for evaluation of the extent of disease. Patients were not considered candidates for the laparoscopic approach when any of the following criteria were present: obvious intraabdominal metastases; bulky uterus (greater than $12 \mathrm{~cm}$ or such that vaginal removal of the uterus might require morcellation); or severe hip conditions that precludes the dorsal lithotomy position. Pelvic lymphadenectomy consisted of removing lymphatic tissue from the mid-portion of the common iliac artery to the circumflex iliac vein, from the mid-portion of the psoas muscle to the hypogastric vessels, and from the obturator fossa anterior to the obturator nerves. Para-aortic lymphadenectomy consisted of removal of nodal tissues over the vena cava and aorta from the mid-right and left common iliac artery to the level of the inferior mesenteric artery and sometimes of the left renal vein.

Operating times were recorded from the first skin incision to the last incision closure. Blood loss was estimated from that collected in the suction device. Complications were defined as any event that required intravenous medications, blood transfusion, surgical procedure, interventional radiology, or major rehabilitation for treatment. Intraoperative hemorrhage was defined as blood loss exceeding 1,000 mL, or requiring a blood transfusion. Active bleeding with symptomatic anemia and hemoglobin less than $8 \mathrm{~g} / \mathrm{dL}$ was considered the criteria for blood transfusions.

We recorded parameters including patient age, parity, BMI, mean operation time, duration of follow up, postoperative adjuvant treatment, estimated blood loss, intraoperative and postoperative blood transfusions, intraoperative and postoperative complications, postoperative hospitalization, International Federation of Gynecology and Obstetrics (FIGO) surgical stage, histopathologic type, tumor grade, number of lymph nodes yielded, tumor size, and recurrence.

Statistical analysis was performed using SPSS ver. 12.0 (SPSS Inc., Chicago, IL, USA). Clinical and pathologic factors were compared between two groups with Pearson's $\chi^{2}$ test or Fisher's exact test for categorical data, and the Student $t$-test and Mann-Whitney $U$ statistic for continuous data according to normality. Progression-free (PFS) and overall survival (OS) were estimated by the Kaplan-Meier method. Differences between survival curves were analyzed using the log-rank test. A p-value less than 0.05 was considered statistically significant.

\section{RESULTS}

Of the 104 patients who were surgically staged with endometrial cancer, 70 were treated by laparotomy and 34 were treated laparoscopically. The mean age of the patients was 51 years in both groups. There were no significant differences in age, parity, mean follow-up, postoperative adjuvant treatment, or recurrence (Table 1). However, in the laparoscopy group, the operation time was longer than that of the laparotomy group $(227.0 \pm 28.8$ vs. $208.1 \pm 46.4$ minutes, $\mathrm{p}=$ 0.032) (Table 1). The mean hospitalization period and estimated blood loss were significantly greater in the laparotomy group than the laparoscopic group (postoperative hospitalization, 23.3 vs. 16.4 days, $\mathrm{p}<0.001$; estimated blood loss, 
Table 1. Clinical characteristics

\begin{tabular}{lccc}
\hline \multicolumn{1}{c}{ Characteristics } & Laparoscopy $(\mathrm{N}=34)$ & Laparotomy $(\mathrm{N}=70)$ & p-value \\
\hline Mean age (yr) & $51 . \pm 18.5$ & $51.0 \pm 9.1$ & $\mathrm{NS}$ \\
Mean parity & $2.3 \pm 1.4$ & $2.0 \pm 1.2$ & $\mathrm{NS}$ \\
Mean BMI $\left(\mathrm{kg} / \mathrm{m}^{2}\right)$ & $23.6 \pm 2.8$ & $25.7 \pm 3.9$ & 0.005 \\
Mean operation time (min) & $227.0 \pm 28.8$ & $208.1 \pm 46.4$ & 0.032 \\
Estimated blood loss (mL) & $230.3 \pm 92.4$ & $301.9 \pm 156.3$ & 0.015 \\
Mean hospitalization (day) & $16.4 \pm 6.7$ & $23.3 \pm 5.6$ & $<0.001$ \\
Mean follow-up (mo) & $20.0 \pm 12.5$ & $24.6 \pm 13.7$ & $\mathrm{NS}$ \\
Adjuvant treatment & $11(32.4)$ & $27(38.6)$ & $\mathrm{NS}$ \\
Recurrence & - & $5(7.1)$ & $\mathrm{NS}$ \\
Death & - & $3(4.3)$ & $\mathrm{NS}$ \\
\hline
\end{tabular}

Values are presented as mean $\pm \mathrm{SD}$ or number (\%).

NS: not significant.

Table 2. Histopathologic characteristics

\begin{tabular}{lccc}
\hline \multicolumn{1}{c}{ Characteristics } & $\begin{array}{c}\text { Laparoscopy } \\
(\mathrm{N}=34)\end{array}$ & $\begin{array}{c}\text { Laparotomy } \\
(\mathrm{N}=70)\end{array}$ & p-value \\
\hline $\begin{array}{l}\text { FIGO stage } \\
\text { (revised in 2009) }\end{array}$ & & & NS \\
I & $26(76.5)$ & $52(74.3)$ & \\
IA & 22 & 45 & \\
IB & 4 & 7 & \\
II & $2(5.9)$ & $10(14.3)$ & \\
III & $6(17.6)$ & $8(11.3)$ & \\
IIIA & - & 2 & \\
IIIB & - & - & \\
IIIC1 & 2 & 2 & \\
IIIC2 & 4 & 4 & \\
Histology & & & NS \\
Endometrioid & $30(88.2)$ & $57(81.4)$ & \\
Non-endometrioid & $4(11.8)$ & $13(18.6)$ & \\
Serous & 2 & 1 & \\
Mixed & 2 & 12 & \\
Grade & & & NS \\
1 & $26(76.5)$ & $44(62.9)$ & \\
2 & $4(11.8)$ & $15(21.4)$ & \\
3 & $4(11.8)$ & $11(15.7)$ & \\
Mean no. of pelvic LNs & $20.8 \pm 8.6$ & $17.2 \pm 9.4$ & NS \\
Mean no. of para-aortic LNs & $9.2 \pm 5.2$ & $8.5 \pm 5.4$ & NS \\
Mean tumor size (cm) & $3.4 \pm 2.4$ & $3.5 \pm 2.6$ & NS \\
\hline
\end{tabular}

Values are presented as mean \pm SD or number (\%).

FIGO: International Federation of Gynecology and Obstetrics, LNs: lymph nodes, NS: not significant.

$301.9 \pm 156.3$ vs. $230.3 \pm 92.4 \mathrm{~mL}, \mathrm{p}=0.015)$ (Table 1$)$.

The histopathologic type, grade, and mean tumor size showed no differences between the two groups (Table 2). The mean numbers of pelvic and para-aortic lymph nodes retrieved were 20.8 and 9.1 in the laparoscopy group and 17.2 and 8.5 in the laparotomy group, respectively. There was no statistically significant difference in the numbers of lymph nodes retrieved between the 2 groups (pelvic lymph nodes, $\mathrm{p}=$ 0.062; para-aortic lymph nodes, $\mathrm{p}=0.554$ ) (Table 2 ).
Table 3. Perioperative complications

\begin{tabular}{lccc}
\hline & $\begin{array}{c}\text { Laparoscopy } \\
(\mathrm{N}=34)\end{array}$ & $\begin{array}{c}\text { Laparotomy } \\
(\mathrm{N}=70)\end{array}$ & p-value \\
\hline Intraoperative complications & $1(2.9)$ & $3(4.3)$ & NS \\
Ureteral injury & - & 2 & \\
Bladder injury & - & 1 & \\
Great vessel injury & 1 & - & \\
Postoperative complications & $4(11.8)$ & $8(11.4)$ & NS \\
Lymphocyst & 4 & 7 & \\
Ileus & - & 1 & \\
Blood transfusion & - & $4(11.8)$ & NS \\
\hline
\end{tabular}

Values are presented as number (\%).

NS: not significant.

Table 4. Site of recurrence

\begin{tabular}{lcc}
\hline & Laparoscopy $(\mathrm{N}=34)$ & Laparotomy $(\mathrm{N}=70)$ \\
\hline No recurrence & 34 & 65 \\
Recurrence & 0 & 5 \\
Pelvic & 0 & 1 \\
Extra-pelvic & 0 & 4 \\
Peritoneum & 0 & 2 \\
Distant & 0 & 1 \\
Multiple & 0 & 1 \\
\hline
\end{tabular}

There was no significant difference in intraoperative or postoperative complications and blood transfusion between the two groups (Table 3). Four patients in the laparotomy group were transfused during surgery, depending on the operator and anesthesiologist's decision. The incidence of intraoperative or postoperative complications was $15.7 \%$ and $14.7 \%$ in the laparotomy and laparoscopy groups, respectively. With regard to intraoperative complications, there was 1 case of great vessel injury in the laparoscopy group, and 2 cases of ureteral injury and 1 case of bladder injury in the laparotomy group. There were 4 cases of lymphocysts in the laparoscopy group compared with 7 in the laparotomy group. No incisional hernias and no case of port-site metastasis have been detected. 
A

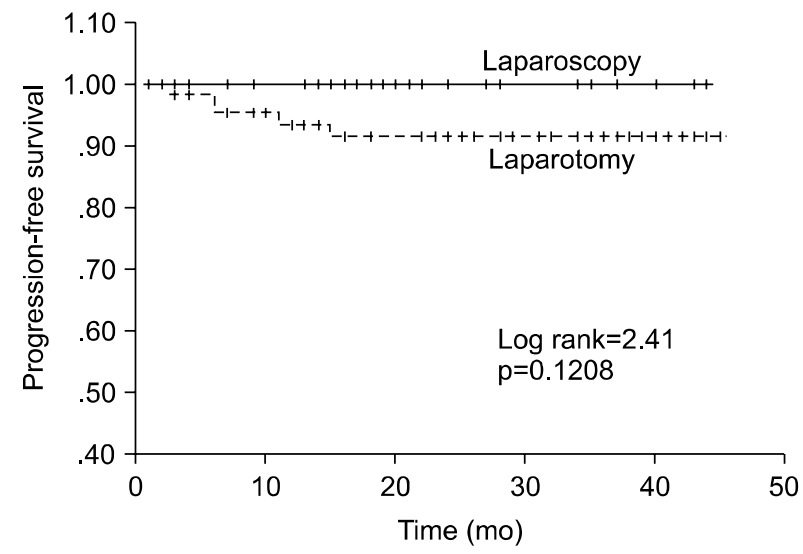

B

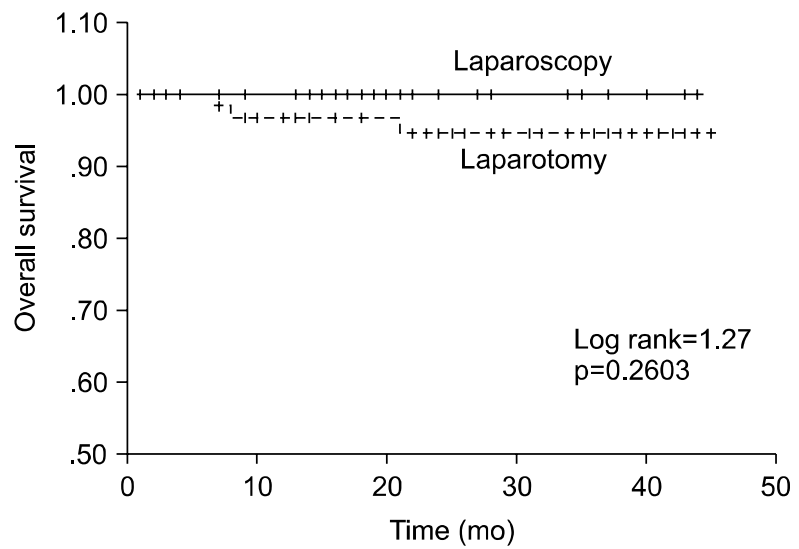

Fig. 1. Progression-free (A) and overall (B) survivals showed no statistically significant differences between the two groups.

Sites of recurrence are listed in Table 4. The mean follow-up time was 25 months (range, 12 to 45 months). During the follow-up time, there was no recurrence in the laparoscopy group. Five patients who received laparotomy experienced disease recurrence; 1 had pelvic recurrence, 2 had peritoneal disease, 1 had multiple lesions, and 1 had distant metastasis. Three patients died of disease.

No statistically significant difference was found between the two groups when PFS $(p=0.1208)$ and OS $(p=0.2603)$ were compared (Fig. 1).

\section{DISSCUSION}

Since the first report describing laparoscopic surgical staging of early endometrial cancer, laparoscopic surgery has been replaced open surgery for early endometrial cancer over the last decade. ${ }^{3-12}$ Disadvantages of the laparoscopic approach include a two-dimensional view, dependence on assistance skill, decreased range of motion and degree of freedom of instruments, and ergonomic limitations to the surgeon. Despite these temporary disadvantages, with the proper training, surgeons are able to adapt to this method of surgery.

In 2009, the GOG reported the results of the LAP2 study, the largest randomized trial ever performed in endometrial cancer. ${ }^{11,13}$ As expected, laparoscopic surgical staging is characterized by fewer postoperative moderate or severe adverse events, shorter hospitalization, longer operative time, and quicker return to normal daily activities. ${ }^{11}$ It is reported that the quality-of-life analyses did not show major significant differences between the two groups. The time to return to work and body image showed only moderate differences, which disappeared 6 months after surgery. ${ }^{13}$

Although several authors have reported on the feasibility and safety of the laparoscopic surgery in early-stage endometrial cancer compared with open surgery, there is no general agreement as to whether laparoscopic treatment for endometrial cancer is comparable to laparotomy in terms of sur- vival, because of lack of sufficient survival data. ${ }^{9-25}$ Recently, Walker et al presented initial survival data from the LAP2 study conducted by the GOG at the 41st annual meeting of the SGO. ${ }^{12}$ The authors showed that the 3-year overall survival was $89.8 \%$ in the laparoscopy group and $89.9 \%$ in the laparotomy group. They suggested that laparoscopic surgical staging is safe, feasible, results in fewer complications, has shorter hospital stay, and should be considered a standard of care for uterine cancer. ${ }^{12}$ However, to date, there has been minimal research regarding the survival benefit of laparoscopic surgery for endometrial cancer, except for the LAP2 trial.

In the current study, there was no statistically significant difference in the numbers of lymph nodes retrieved, intraoperative or postoperative complications, and survival rates between the two groups. The operation time was significantly longer for patients undergoing laparoscopy when compared to laparotomy. It seems that the only weakness in laparoscopic staging for endometrial cancer is the longer operation time. However, several recent studies showed that there is a tendency for shorter laparoscopic operation time and increased use of the laparoscopic approach for endometrial cancer as surgical skills are progressing. ${ }^{26,27}$ The preoperative and postoperative hemoglobin changes were significantly greater in the laparotomy group than the laparoscopic group. It seems that more precise and less bleeding in surgery is possible due to better visualization, and ability to obtain access to spaces more easily due to insufflation of the abdomen. The numbers of nights in the hospital and complications have been reduced for laparoscopic surgery, with more rapid recovery relative to open surgery. ${ }^{23-28}$ The postoperative hospitalization was significantly shorter for patients in the laparoscopy group, but it was longer than that reported in the United States. After lymphadenectomy, the drainage tubes are usually inserted to examine postoperative bleeding. Drainage tubes are removed at the discretion of individual clinicians, which may explain the extended hospitalization. In Korea, most patients wish to be discharged after complete recovery (i.e., removal of the lym- 
phatic fluid drainage tubes). Therefore, the length of stay may be dependent on the complete removal of drainage tubes and stitches. Moreover, medical insurance companies in Korea fully reimburse medical costs, and patients are less concerned about the duration of hospital stay for cancer patients., ${ }^{5,26}$

A potential limitation of our study is the small number of cases and selection bias, which is inherent in any retrospective study. In the current study, patients were non-randomly assigned to laparoscopy or laparotomy. This selection bias may influence our results and, in the present study, the mean BMI was higher in the laparotomy group than the laparoscopy group ( $\mathrm{p}=0.005$ ). The short follow-up is another potential limitation and limits clear conclusions. Despite these limitations, this study suggests the possible survival benefit of laparoscopic treatment for endometrial cancer, and supports the positive results of the LAP2 trial.

In summary, it seems that laparoscopic surgical staging operation is a safe and effective therapeutic procedure for management of endometrial cancer, with an acceptable morbidity compared to the laparotomic approach, and is characterized by far less blood loss and shorter postoperative hospitalization time. Recently, some reports demonstrated that robotic surgery is superior to laparoscopy in surgical staging of endometrial cancer. ${ }^{29,30}$ However, the high cost limits universal use. Further multicenter randomized trials with longer follow-up should be necessary to evaluate the overall oncologic outcomes of this procedure.

\section{CONFLICT OF INTEREST}

No potential conflict of interest relevant to this article was reported.

\section{REFERENCES}

1. Jemal A, Siegel R, Ward E, Murray T, Xu J, Thun MJ. Cancer statistics, 2007. CA Cancer J Clin 2007; 57: 43-66.

2. Sorosky JI. Endometrial cancer. Obstet Gynecol 2008; 111: 436-47.

3. Childers JM, Surwit EA. Combined laparoscopic and vaginal surgery for the management of two cases of stage I endometrial cancer. Gynecol Oncol 1992; 45: 46-51.

4. Eltabbakh GH, Shamonki MI, Moody JM, Garafano LL. Laparoscopy as the primary modality for the treatment of women with endometrial carcinoma. Cancer 2001; 91: 378-87.

5. Kim DY, Kim MK, Kim JH, Suh DS, Kim YM, Kim YT, et al. Laparoscopic-assisted vaginal hysterectomy versus abdominal hysterectomy in patients with stage I and II endometrial cancer. Int J Gynecol Cancer 2005; 15: 932-7.

6. Ghezzi F, Cromi A, Bergamini V, Uccella S, Beretta P, Franchi $M$, et al. Laparoscopic management of endometrial cancer in nonobese and obese women: a consecutive series. J Minim Invasive Gynecol 2006; 13: 269-75.

7. Yu CK, Cutner A, Mould T, Olaitan A. Total laparoscopic hysterectomy as a primary surgical treatment for endometrial cancer in morbidly obese women. BJOG 2005; 112: 115-7.

8. Scribner DR Jr, Walker JL, Johnson GA, McMeekin SD, Gold MA, Mannel RS. Surgical management of early-stage endo- metrial cancer in the elderly: is laparoscopy feasible? Gynecol Oncol 2001; 83: 563-8.

9. Palomba S, Falbo A, Mocciaro R, Russo T, Zullo F. Laparoscopic treatment for endometrial cancer: a meta-analysis of randomized controlled trials (RCTs). Gynecol Oncol 2009; 112: 415-21.

10. Ju W, Myung SK, Kim Y, Choi HJ, Kim SC. Comparison of laparoscopy and laparotomy for management of endometrial carcinoma: a meta-analysis. Int J Gynecol Cancer 2009; 19: 400-6.

11. Walker JL, Piedmonte MR, Spirtos NM, Eisenkop SM, Schlaerth JB, Mannel RS, et al. Laparoscopy compared with laparotomy for comprehensive surgical staging of uterine cancer: Gynecologic Oncology Group Study LAP2. J Clin Oncol 2009; 27: 5331-6.

12. Walker JL, Piedmonte MR, Spirtos NM, Eisenkop SM, Spiegel G, Mannel RS, et al. Recurrence and survival after randomization to laparoscopy versus laparotomy for comprehensive surgical staging of uterine cancer: Gynecologic Oncology Group Study LAP2. 2010 SGO 41st Annual Meeting, Abstract LBA1.

13. Kornblith AB, Huang HQ, Walker JL, Spirtos NM, Rotmensch J, Cella D. Quality of life of patients with endometrial cancer undergoing laparoscopic international federation of gynecology and obstetrics staging compared with laparotomy: a Gynecologic Oncology Group study. J Clin Oncol 2009; 27: 5337-42.

14. Obermair A, Manolitsas TP, Leung Y, Hammond IG, McCartney AJ Total laparoscopic hysterectomy versus total abdominal hysterectomy for obese women with endometrial cancer. Int J Gynecol Cancer 2005; 15: 319-24.

15. Barakat RR, Lev G, Hummer AJ, Sonoda Y, Chi DS, Alektiar $\mathrm{KM}$, et al. Twelve-year experience in the management of endometrial cancer: a change in surgical and postoperative radiation approaches. Gynecol Oncol 2007; 105: 150-6.

16. Obermair A, Manolitsas TP, Leung Y, Hammond IG, McCartney AJ. Total laparoscopic hysterectomy for endometrial cancer: patterns of recurrence and survival. Gynecol Oncol 2004; 92: 789-93.

17. Kuoppala T, Tomas E, Heinonen PK. Clinical outcome and complications of laparoscopic surgery compared with traditional surgery in women with endometrial cancer. Arch Gynecol Obstet 2004; 270: 25-30.

18. Holub Z, Jabor A, Bartos P, Eim J, Urbanek S, Pivovarnikova R. Laparoscopic surgery for endometrial cancer: long-term results of a multicentric study. Eur J Gynaecol Oncol 2002; 23: 305-10.

19. Fram KM. Laparoscopically assisted vaginal hysterectomy versus abdominal hysterectomy in stage I endometrial cancer. Int J Gynecol Cancer 2002; 12: 57-61.

20. Malur S, Possover M, Michels W, Schneider A. Laparoscopic-assisted vaginal versus abdominal surgery in patients with endometrial cancer: a prospective randomized trial. Gynecol Oncol 2001; 80: 239-44.

21. Gemignani ML, Curtin JP, Zelmanovich J, Patel DA, Venkatraman E, Barakat RR. Laparoscopic-assisted vaginal hysterectomy for endometrial cancer: clinical outcomes and hospital charges. Gynecol Oncol 1999; 73: 5-11.

22. Boike GM, Elfstrand EP, DelPriore G, Schumock D, Holley HS, Lurain JR. Laparoscopically assisted vaginal hysterectomy in a university hospital: report of 82 cases and comparison with abdominal and vaginal hysterectomy. Am J Obstet Gynecol 1993; 168: 1690-7.

23. Kalogiannidis I, Lambrechts S, Amant F, Neven P, Van Gorp T, Vergote I. Laparoscopy-assisted vaginal hysterectomy compared with abdominal hysterectomy in clinical stage I endometrial cancer: safety, recurrence, and long-term outcome. Am J Obstet Gynecol 2007; 196: 248. e1-8.

24. Malzoni M, Tinelli R, Cosentino F, Perone C, Rasile M, Iuzzolino D, et al. Total laparoscopic hysterectomy versus abdominal hysterectomy with lymphadenectomy for early-stage endometrial cancer: a prospective randomized study. Gynecol 
Oncol 2009; 112: 126-33.

25. Zullo F, Palomba S, Falbo A, Russo T, Mocciaro R, Tartaglia E, et al. Laparoscopic surgery vs laparotomy for early stage endometrial cancer: long-term data of a randomized controlled trial. Am J Obstet Gynecol 2009; 200: 296. e1-9.

26. Cho YH, Kim DY, Kim JH, Kim YM, Kim YT, Nam JH. Laparoscopic management of early uterine cancer: 10-year experience in Asan Medical Center. Gynecol Oncol 2007; 106: 585-90.

27. Hahn HS, Kim HJ, Yoon SG, Kim WC, Choi HJ, Kim HS, et al. Laparoscopy-assisted vaginal versus abdominal hysterectomy in endometrial cancer. Int J Gynecol Cancer 2010; 20: 102-9.

28. Magrina JF. Outcomes of laparoscopic treatment for endometrial cancer. Curr Opin Obstet Gynecol 2005; 17: 343-6.

29. Lowe MP, Johnson PR, Kamelle SA, Kumar S, Chamberlain DH, Tillmanns TD. A multiinstitutional experience with robotic-assisted hysterectomy with staging for endometrial cancer. Obstet Gynecol 2009; 114: 236-43.

30. Cardenas-Goicoechea J, Adams S, Bhat SB, Randall TC. Surgical outcomes of robotic-assisted surgical staging for endometrial cancer are equivalent to traditional laparoscopic staging at a minimally invasive surgical center. Gynecol Oncol 2010; 117: 224-8. 